\title{
Desorption Ionization by Charge Exchange (DICE) for Sample Analysis under Ambient Conditions by Mass Spectrometry
}

\author{
Chang-Ching Chan, a,b Mark S. Bolgar, ${ }^{\mathrm{b}}$ Scott A. Miller, ${ }^{\mathrm{b}}$ and \\ Athula B. Attygalle ${ }^{a}$ \\ a Center for Mass Spectrometry, Department of Chemistry, Chemical Biology, and Biomedical Engineering, \\ Stevens Institute of Technology, Hoboken, New Jersey, USA \\ ${ }^{\mathrm{b}}$ Bristol-Myers Squibb Analytical Research and Development, New Brunswick, New Jersey, USA
}

\begin{abstract}
An ambient pressure ionization technique for mass spectrometric analysis of substances present on solid surfaces was developed. A nebulized spray containing molecular ions of a solvent such as toluene can be generated by passing the solvent through a stainless steel capillary held at a high voltage. When the stream of charged droplets produced in this way is directed onto a solid surface, the analytes present on the surface are desorbed and ionized by a charge exchange process. This technique was shown to desorb and generate positively charged molecular ions from compounds that are not readily ionized by some other ambient methods, under positive-ion generation mode. For example, intense signals representing radical cations of 1,4-hydroquinone, limonene, thymol, and several other compounds were observed when the analytes were deposited on a metal surface and exposed to a toluene spray nebulized from the metal capillary maintained at a potential of about $+5 \mathrm{kV}$. In contrast, when the same samples were exposed to a spray of water/methanol/formic acid under customary DESI-like (positive-ion mode) conditions, no peaks representing the analytes were observed. (J Am Soc Mass Spectrom 2010, 21, 1554-1560) (C) 2010 American Society for Mass Spectrometry
\end{abstract}

$\mathrm{W}$ ith the introduction of ambient desorption ionization techniques such as desorption electrospray ionization (DESI) and direct analysis in real time (DART), a range of compounds, including proteins [1, 2], explosives [3, 4], polymers [5], pharmaceuticals [6], and metabolites $[3,4]$ are now amenable to atmospheric-pressure ionization mass spectrometry with little or no sample preparation. The DESI technique has been extended even to sample imaging [7]. Despite these major breakthroughs, both techniques bear a few limitations. The DART technique can be applied primarily to low-molecular-weight samples $(<\sim 1 \mathrm{kDa})$, and has a limited dynamic range for quantitative determinations $[4,8]$. In addition, a costly ion source dedicated to the DART technique is required to apply this method. In contrast, the DESI procedure has a higher dynamic range and molecular weight limits [9]. Moreover, this procedure can be performed using a conventional ESI probe commonly available on atmospheric pressure-ionization mass spectrometers. However, the conventional DESI procedure does not efficiently ionize many samples of low polarity [10]. The detection of trace amounts of compounds of low proton

Address reprint requests to Professor A. Attygalle, Center for Mass Spectrometry, Department of Chemistry and Chemical Biology, Stevens Institute of Technology, Castle Point on Hudson, Hoboken, NJ 07030, USA. E-mail: athula.attygalle@stevens.edu affinity and low acidity, such as cholesterol, can be achieved only by reactive DESI procedures [11]. Although the direct DESI technique has been reported to ionize some highly conjugated nonpolar compounds such as lycopene [12], better detection of saturated hydrocarbons is achieved only under dischargeinduced oxidation conditions [13]. However, dischargeinduced oxidation is nonspecific, therefore unsuitable for structure elucidation work [13].

In this report, we describe a technique that can be easily incorporated into an existing DESI or ESI sources. In this way, positively charged molecular ions can be generated under ambient conditions from a wide range of compounds of different polarity. To distinguish this technique from DESI and regular ESI, we propose to call it desorption ionization by charge exchange (DICE). Although DICE uses a DESI or an ESI source, this new technique has a different ionization mechanism than those of ESI-related procedures. In ESI-related desorption techniques, the analytes are cationized by either forming the protonated species or the metal adducts. In contrast, DICE forms gaseous ions through a charge exchange process between the neutral analytes and the molecular ions of the spray reagent. We postulate that the molecular ions of the spray reagent that is required for DICE are formed by an electrochemical oxidation process that occurs at the metal-solvent interface of the 
capillary needle, an observation that Van Berkel et al. had previously reported for other compounds [14].

DICE shares some similarities with the previously described desorption atmospheric-pressure chemicalionization (DAPCI) procedure [15], and desorption atmospheric-pressure photoionization (DAPPI) technique [10], both of which use a dopant such as toluene to assist the ion formation. In DAPCI, a reagent gas (e.g., nitrogen, methanol, or toluene vapor) is first ionized by a corona discharge and used to desorb and ionize samples from surfaces under ambient conditions [15]. DAPPI, on the other hand, desorbs samples from ambient surfaces by first exposing analytes to the hot solvent vapor (e.g., toluene) generated from a heated nebulizer microchip, and then ionizes the gaseous analytes by interacting with energetic photons from a krypton lamp [10]. Unlike DAPCI or DAPPI procedures, the DICE technique described here uses the reagent ions (i.e., molecular ions of toluene) formed in solution before the nebulization step.

\section{Experimental}

\section{Mass Spectrometry}

Except for a few experiments described below, all other data were acquired on a Waters Quattro Micro tandem quadrupole mass spectrometer (Milford, MA, USA). The configuration of the DICE ion source is illustrated in Figure 1. The front glass panel of the Quattro Micro instrument that isolates the ion source region from the outside atmosphere was replaced with a polycarbonate panel, cut to similar dimensions ( 3.75 in. $\times 3.75$ in.). A rectangular slit $(\sim 0.25$ in. $\times 0.15$ in. $)$ was cut in the panel to introduce the target probe containing analytes. Experiments were conducted also on a linear ion trap (LTQ) (Thermo Fisher, Waltham, MA) mass spectrometer using either a fused-silica or a stainless-steel spray capillary to investigate the electrochemical oxidation process that toluene undergoes.



Figure 1. Schematic of the DICE ion source.

\section{Instrumental Parameters and Conditions}

Toluene was infused at a flow rate of $50 \mu \mathrm{L} / \mathrm{min}$ through the ESI metal capillary $(100 \mu \mathrm{m})$ which was held at $5.0 \mathrm{kV}$ for DICE experiments. For DESI-type experiments, the reagent spray used was a solution of $0.1 \%$ formic acid in 50\%-70\% water: $30 \%-50 \%$ methanol, or $0.1 \%$ formic acid in $70 \%$ water: $30 \%$ acetonitrile at a flow rate between $10 \mu \mathrm{L} / \mathrm{min}$ to $15 \mu \mathrm{L} / \mathrm{min}$. For the Quattro Micro instrument, the desolvation gas (nitrogen) flow rate and temperature were set at $450 \mathrm{~L} / \mathrm{h}$ and $350{ }^{\circ} \mathrm{C}$, respectively. The cone voltage was set at $25 \mathrm{~V}$, and no cone gas was applied. The source was maintained at $125^{\circ} \mathrm{C}$. For the linear ion trap instrument (Thermo Finnigan LTQ), the spray voltage was set at 5-6 kV and the capillary (fused silica, $100 \mu \mathrm{m}$ ) temperature was maintained at $150{ }^{\circ} \mathrm{C}$. The sheath gas, sweep gas, and auxiliary gas flow rates were set to 30,5 , and 10 arbitrary units, respectively.

\section{Materials}

Vitamin K, cholesterol, and estradiol were purchased from Alfa Aesar (Wardhill, MA , USA). 2-Naphthol and DL- $\alpha$-tocopherol were purchased from TCI America (Portland, OR, USA). 1,4-Hydroquinone, anthracene, p-aminobenzoic acid, retinol, limonene, thymol, phenyl acetaldehyde, farnesyl acetate, 4-chlorophenol, 4-iodophenol, di- $n$-hexyl ketone, $p$-cresol, benzaldehyde, $\gamma$ terpinene, $\beta$-pinene oxide, $\beta$-caryophyllene, fluoranthene, salicylaldoxime, and ferrocene were purchased from Sigma-Aldrich (St. Louis, MO, USA). Formic acid, hexane, and toluene $(99.8 \%)$ were purchased from EMD Chemical (Gibbstown, NJ, USA). Methanol and acetonitrile were purchased from J. T. Baker (Philipsburg, NJ, USA). Water purified using a Milli-Q purification system (Billerica, MA, USA) was used for all experiments. A piece of braided stainless steel wire $(\sim 1 / 8$ in. o.d. $X$ 2 in.), obtained from OOK (Miami, FL, USA), was used as the target surface to which samples were applied. The target surface was cleaned with methanol before use. The polycarbonate sheet that was used in place of the front glass window of the ion source was purchased from SABIC-IP (Pittsfield, MA, USA).

\section{Sample Preparations}

The concentration of the stock solutions of individual analytes ranged between 7 and $0.07 \mathrm{mg} / \mathrm{mL}$. Estradiol and 1,4-hydroquinone were dissolved in methanol, and other samples such as vitamin K, cholesterol, 2-naphthol, anthracene, vitamin A, $\alpha$-tocopherol, $p$-aminobenzoic acid, limonene, thymol, phenylacetaldehyde, farnesyl acetate, 4-chlorophenol, 4-iodophenol, di- $n$-hexyl ketone, $p$-cresol, benzaldehyde, $\gamma$-terpinene, $\beta$-pinene oxide, $\beta$-caryophyllene, fluoranthene, salicylaldoxime, and ferrocene were dissolved in toluene. The amount of each analyte deposited on the target surface (braided stainless steel wire) ranged from 1 to $100 \mu \mathrm{g}$. To deposit 
$1 \mu \mathrm{g}$ of analyte on the target surface, for example, $15 \mu \mathrm{L}$ of the $0.07 \mathrm{mg} / \mathrm{mL}$ stock solution was pipetted and deposited on the surface of area $\sim 40 \mathrm{~mm}^{2}$ (the surface area was calculated by assuming that the braided wire has a surface area similar to that of a solid rod). The sample on the surface was air-dried at room temperature for 0.5 to $2 \mathrm{~min}$ before subjecting the deposits to desorption-ionization experiments.

\section{Sample Introduction}

For all DESI and DICE experiments, the target probe was inserted through the open slot in the polycarbonate source window panel. The probe wire was held $\sim 1$ to $2 \mathrm{~cm}$ away from the ESI capillary tip and $\sim 2$ to $3 \mathrm{~cm}$ away from the entrance cone. The suggested incident $(\alpha)$ and collection $(\beta)$ angles for DESI were $55^{\circ}$ and $10^{\circ}$, respectively, for optimal signal intensity [16, 17]. However, the $\alpha$ and $\beta$ angles for all experiments performed in this work had to be set at $\alpha \sim \beta \sim 80^{\circ}$ because of physical constraints of the instrument. Hence, the DESI data presented here were collected under non-optimized conditions. A clean wire was used as the control to generate background spectra.

\section{Results and Discussion}

The DESI technique is widely used in mass spectrometry to desorb and ionize analytes from solid substrates [16]. This technique, which uses a fine spray of charged droplets generated by an electrospray probe from polar solvents, shares many common characteristics as the ESI technique. One of such characteristic is that DESI usually ionizes neutral and nonpolar compounds less effectively or not at all [10]. For example, a sample of 1,4-hydroquinone, limonene, or thymol deposited on a metal surface failed to show any sample related peaks when a spectrum was recorded by spraying a mixture of water/methanol/formic acid through the electrospray capillary under DESI conditions in positive ion mode (Figure 2a, c, e). In contrast, we found intense signals for the positively charged molecular ions when toluene was used as the spray reagent (Figure $2 b, d, f$ ). We hypothesize that under positive-ion conditions, when toluene is passed through the charged capillary tube, molecular ions of toluene are generated by an electrochemical oxidation process, which imparts a net charge to the nebulized droplets. To further investigate the hypothesis that the toluene droplets are charged before leaving the capillary tip, we carefully observed and compared the shape of the toluene droplets at the first onset of the spray to that from polar solvents at the tip of the capillary held at high voltages. When no voltage and pneumatic assistance are applied to the capillary, the initial droplets emanating from polar solvents are somewhat spherical in shape. Once the capillary voltage required for electrospray is applied, the droplets become elongated. This deformation from the initial spherical shape, which occurs at about 2 to 3 $\mathrm{kV}$, is an indication of the formation of charged droplets (so-called Taylor cone formation) [18]. With toluene, a similar the deformation could be observed at 4.5 to $5 \mathrm{kV}$ (of course, this can be observed only if the nebulization gas is turned off). Presumably, this observation indicates that the droplets formed from toluene, with the capillary voltage on, are charged as a consequence of the electrochemical oxidation that takes place on the capillary surface as described by Van Berkel and coworkers [14]. The magnitude of the capillary voltage plays an important role in the production of toluene molecular ions. For example, the peak at $m / z$ 92, for the molecular ion of toluene, was observed only when the high voltage (about $5 \mathrm{kV}$ ) was applied to the stainless steel spray capillary. At capillary voltages lower than $4.5 \mathrm{kV}$, no molecular ions of toluene were detected. Similar results were obtained when the LTQ equipped with a stainless steel capillary was used. However, when the capillary was switched to one that has similar dimensions but made of fused silica, no peak at $\mathrm{m} / \mathrm{z} 92$ was observed (data not shown) at a capillary voltage $(5-6 \mathrm{kV})$. These observations point out that the electro-

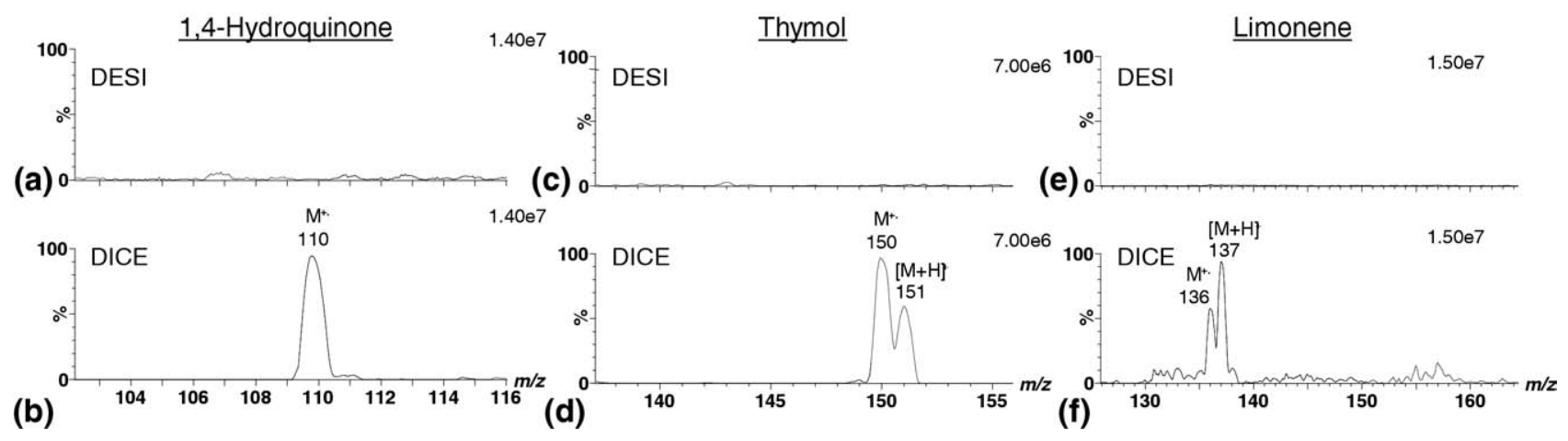

Figure 2. Background-subtracted mass spectra (average of at least 3 MS scans) obtained by spraying water/methanol/formic acid (DESI capillary voltage $3.5 \mathrm{kV})(\mathbf{a}),(\mathbf{c}),(\mathbf{e})$, or toluene (DICE capillary voltage $5 \mathrm{kV})(\mathbf{b}),(\mathbf{d})$, (f) through the ESI capillary tube, on 1,4-hydroquinone (nominal mass 110), thymol (nominal mass 150), and limonene (nominal mass 136) deposited on a metal wire. The amount of 1,4-hydroquinone on the target surface was estimated to be $2.5 \mu \mathrm{g} / \mathrm{mm}^{2}$ while thymol and limonene was $\sim 250 \mathrm{ng} / \mathrm{mm}^{2}$. 
chemical oxidation at the metal surface (which does not take place with fused silica) plays an important role in the ionization process. In addition to the electrochemical oxidation mechanism, some gaseous molecular ions of toluene might also be formed by the corona discharge at the capillary tips.

The molecular ions (generated by both electrochemical oxidation and corona discharge mechanisms) when directed onto a solid surface deposited with analytes, the substances on the surface were ionized by a charge exchange process (Scheme 1).

To illustrate the versatility of the DICE technique, mass spectra of a wide range of compounds were recorded (Figures S1-S24, which can be found in the electronic version of this article, and Table 1). The DICE technique generated molecular ions from all the compounds listed in Table 1. In some favorable cases, the signal to noise ratio generated by the DICE procedure was better than that obtained by the DESI procedure (however, this is a semi-quantitative estimation because the instrument used for comparison was not an optimized commercial DESI instrument). A limitation of the DICE technique, which in fact is common to all chemical ionization and matrix-based techniques, is the high chemical noise in the low-mass region. To overcome this limitation and to identify peaks specific to a particular sample, background spectra originating from toluene and the target surface impurities were recorded and subtracted from the averaged spectra of the analytes (Figure 3a). Figure $3 b$ and $c$ show the typical background peaks [benzyl cation $(\mathrm{m} / \mathrm{z} 91)$, molecular ion of toluene $(m / z 92)$, protonated toluene $(m / z 93)$, methyl-substituted benzyl ion $(m / z 105)$, molecular ion of xylene $(\mathrm{m} / \mathrm{z} 106)$, and molecular ion of cresol $(\mathrm{m} / \mathrm{z}$ $108)$ ] observed by spraying toluene on a metal target. The peak at $m / z 106$ was due to traces of xylenes present as impurities in toluene. Presumably, the molecular ion of cresol was formed by an oxidation reaction between



Scheme 1. The proposed mechanism of charge exchange by DICE technique illustrated using toluene (spray reagent) and 1,4-hydroquinone (analyte) as an example. the molecular ion of toluene and oxygen in the gaseous phase [19]. The interference from toluene-related peaks becomes less problematic when analytes with high molecular weight are being investigated. Similar observations have been made by Traldi and coworkers from their atmospheric pressure photoionization (APPI) work [19].

The observation of molecular ion peaks for all analytes listed in Table 1 suggests that the main ionization mechanism in the DICE technique is a charge exchange process. However, upon evaluating the DICE spectra in more detail, it became apparent that contributions from other ionization mechanisms are also possible. For example, in the spectrum recorded from cholesterol by the DICE technique, a peak at $m / z 369$ that corresponds to the $\left[\mathrm{MH}-\mathrm{H}_{2} \mathrm{O}\right]^{+}$ion of cholesterol was observed in addition to that of its molecular ion (Figure 4a). A similar peak at $m / z 369$ had been observed previously when cholesterol was analyzed using the DART procedure as reported by Cody [20]. It has been stated that the ionization mechanism of the DART technique is APCI-related [4,9], therefore, the similarity between cholesterol spectra generated by DICE and DART technique suggest that there could be some contributions from the APCI-related ionizations during DICE ionization.

Interestingly, the DESI experiments presented in this work appear to have some dependence on the applied capillary voltage. When the capillary voltage was set at $5 \mathrm{kV}$, a cholesterol spectrum similar to that from the DART [20] procedure was obtained (Figure 4b). However, when the capillary voltage was reduced to $3.5 \mathrm{kV}$, no significant peaks that represent cholesterol were observed (Figure 4c). As such, apparently in the experiments discussed here, the ion generation by DESI process is somewhat enhanced by contributions from the APCI-related mechanisms when the capillary was held at $\sim 5 \mathrm{kV}$. Other examples of this dependence on capillary voltage by DESI are shown in Supplementary Figures S25-S26, where 2-naphthol and $\alpha$-tocopherol undergo a decrease in ion abundance when the capillary voltage was lowered from 5 to $3.5 \mathrm{kV}$.

To distinguish the APCI-related ionization mechanisms from those of the DICE and DESI procedures, anthracene was desorbed and ionized by DICE (Figure 3a) and DESI procedures with the capillary voltage held at $5 \mathrm{kV}$ (Figure 5a), and the spectra obtained were compared with the results obtained from desorption using nitrogen gas only under identical conditions (Figure 5b). The desorption medium that used nitrogen only was considered to mimic the ionization process of the DAPCI. The spectral differences between DICE and APCI-related technique were quite distinctive. The most predominant peak in the DICE spectrum (Figure $3 a)$ was the molecular ion of anthracene $(\mathrm{m} / \mathrm{z} 178)$, whereas the DAPCI method produced primarily a peak at $m / z 179$ for the protonated species (Figure 5b). The DESI technique, on the other hand, generated a spectrum that is similar to that of the DAPCI (Figure 5a and 
Table 1. List of compounds evaluated by DICE technique

\begin{tabular}{|c|c|c|c|}
\hline Compound & $\begin{array}{l}\text { Amount deposited on } \\
\text { surface }\left(\mathrm{ng} / \mathrm{mm}^{2}\right)\end{array}$ & Detected $m / z$ (ion type) & $\begin{array}{c}\text { Approximate } \mathrm{S} / \mathrm{N} \text { intensity ratio of } \\
\text { molecular ion peak }\end{array}$ \\
\hline Vitamin $\mathrm{K}$ & 25 & $450\left(\mathrm{M}^{+\bullet}\right), 451[\mathrm{MH}]^{+}$ & $20: 1$ \\
\hline Cholesterol & 25 & $386\left(\mathrm{M}^{+\bullet}\right), 369\left[\mathrm{MH}-\mathrm{H}_{2} \mathrm{O}\right]^{+}$ & $10: 1$ \\
\hline Estradiol & 100 & $272\left(\mathrm{M}^{+\bullet}\right), 255\left[\mathrm{MH}-\mathrm{H}_{2} \mathrm{O}\right]^{+}$ & $20: 1$ \\
\hline 2-Naphthol & 25 & $144\left(\mathrm{M}^{+\bullet}\right), 145[\mathrm{MH}]^{+}$ & $100: 1$ \\
\hline 1,4-Hydroquinone & 100 & $110\left(\mathrm{M}^{+\bullet}\right)$ & $20: 1$ \\
\hline Anthracene & 25 & $178\left(\mathrm{M}^{+\bullet}\right), 179[\mathrm{MH}]^{+}$ & $100: 1$ \\
\hline Vitamin A & 250 & $286\left(\mathrm{M}^{+\bullet}\right), 269\left[\mathrm{MH}-\mathrm{H}_{2} \mathrm{O}\right]^{+}$ & $20: 1$ \\
\hline DL- $\alpha$-tocopherol & 25 & $430\left(\mathrm{M}^{+\bullet}\right), 431[\mathrm{MH}]^{+}$ & $20: 1$ \\
\hline$p$-Aminobenzoic acid & 25 & $137\left(\mathrm{M}^{+\bullet}\right), 138[\mathrm{MH}]^{+}$ & $20: 1$ \\
\hline Limonene & 250 & $136\left(\mathrm{M}^{+\bullet}\right), 137[\mathrm{MH}]^{+}$ & $10: 1$ \\
\hline Thymol & 250 & $150\left(\mathrm{M}^{+\bullet}\right), 151[\mathrm{MH}]^{+}$ & $100: 1$ \\
\hline Phenylacetaldehyde & 250 & $120\left(\mathrm{M}^{+\bullet}\right), 121[\mathrm{MH}]^{+}$ & $100: 1$ \\
\hline Farnesyl acetate & 250 & $264\left(\mathrm{M}^{+\bullet}\right), 265[\mathrm{MH}]^{+}$ & $10: 1$ \\
\hline 4-Chlorophenol & 250 & $128 / 130\left(\mathrm{M}^{+\bullet}\right)$ & $20: 1$ \\
\hline 4-lodophenol & 250 & $220\left(\mathrm{M}^{+\bullet}\right)$ & $50: 1$ \\
\hline Dihexyl ketone & 250 & $198\left(\mathrm{M}^{+\bullet}\right), 199[\mathrm{MH}]^{+}$ & $20: 1$ \\
\hline$p$-Cresol & 250 & $108\left(\mathrm{M}^{+\bullet}\right)$ & $100: 1$ \\
\hline Benzaldehyde & 250 & $106\left(\mathrm{M}^{+\bullet}\right), 105[\mathrm{MH}]^{+}, 106[\mathrm{MH}]^{+}$ & $10: 1$ \\
\hline$\gamma$-Terpinene & 250 & $136\left(\mathrm{M}^{+\bullet}\right), 137[\mathrm{MH}]^{+}$ & $50: 1$ \\
\hline$\beta$-Pinene oxide & 250 & $152\left(\mathrm{M}^{+\bullet}\right), 153[\mathrm{MH}]^{+}$ & $5: 1$ \\
\hline$\beta$-Caryophyllene & 250 & $204\left(\mathrm{M}^{+} \bullet\right), 205[\mathrm{MH}]^{+}$ & $50: 1$ \\
\hline Fluoranthene & 250 & $202\left(\mathrm{M}^{+} \bullet\right), 203[\mathrm{MH}]^{+}$ & $10: 1$ \\
\hline Salicylaldoxime & 250 & $137\left(\mathrm{M}^{+\bullet}\right), 138[\mathrm{MH}]^{+}$ & $20: 1$ \\
\hline Ferrocene & 250 & $187\left(\mathrm{M}^{+\bullet}\right), 187[\mathrm{MH}]^{+}$ & 100:1 \\
\hline
\end{tabular}

b). It should be noted that, as discussed previously, the data presented in Figure 5a could have some contributions from the APCI-related mechanisms because the capillary was held at high voltage $(5 \mathrm{kV})$. In the DAPCI spectrum (Figure 5b), however, a small peak that corresponds to the molecular ion of anthracene at $\mathrm{m} / \mathrm{z} 178$ was observed, while no molecular ion peak of anthracene was observed in the DESI spectrum (Figure 5a). DAPCI is known to generate neutral metastable species by a gas-phase electrical discharge, e.g., excited-state of ambient nitrogen, oxygen, and water etc., which can act as reagents for chemical ionization $[9,15,21]$. The peak



Figure 3. Positive-ion mass spectra recorded using the DICE technique. Background subtracted mass spectrum of anthracene (a), raw mass spectrum of anthracene with no background subtraction (b), and mass spectrum of blank target surface (c). The amount of anthracene deposited on the target was $2.5 \mu \mathrm{g} / \mathrm{mm}^{2}$. for the molecular ion of anthracene as shown in Figure $5 b$ is probably produced by a reaction between a desorbed analyte and these metastable species. The detection of only a small peak for the molecular ion anthracene at $m / z 178$, Figure $5 b$, suggests that the formation of the molecular ions of analytes by direct corona discharge or interaction with the metastable species in the gaseous phase is not the dominant process under DICE conditions.

Cooks and coworkers had proposed that the main desorption mechanism in DESI can be attributed to a droplet "pick-up" process followed by a subsequent evaporation of droplets that leads to the generation of

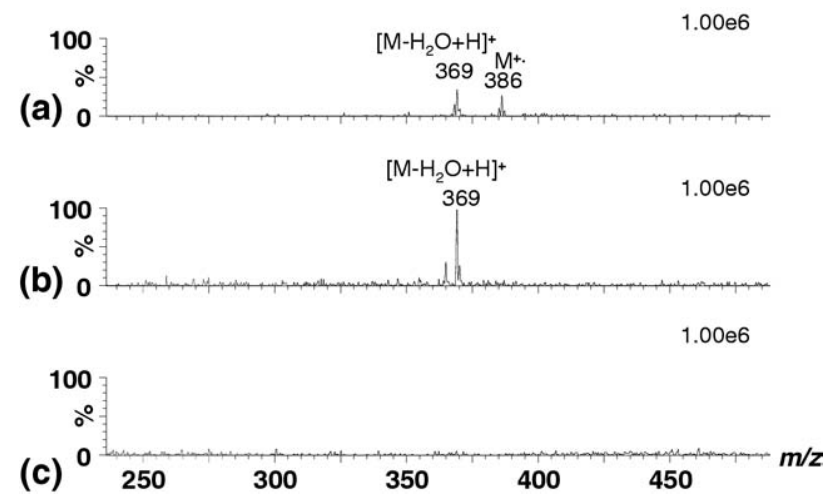

Figure 4. Mass spectra generated by spraying toluene at a capillary voltage of $5 \mathrm{kV}$ by the DICE procedure (a), or water/ acetonitrile/formic by the DESI procedure at a capillary voltage of $5 \mathrm{kV}(\mathbf{b})$, and $3.5 \mathrm{kV}(\mathbf{c})$ on a target deposited with $100 \mathrm{ng} / \mathrm{mm}^{2}$ of cholesterol. The spectra have been normalized to display the same ion intensity scale on the y-axis.. 
(a)

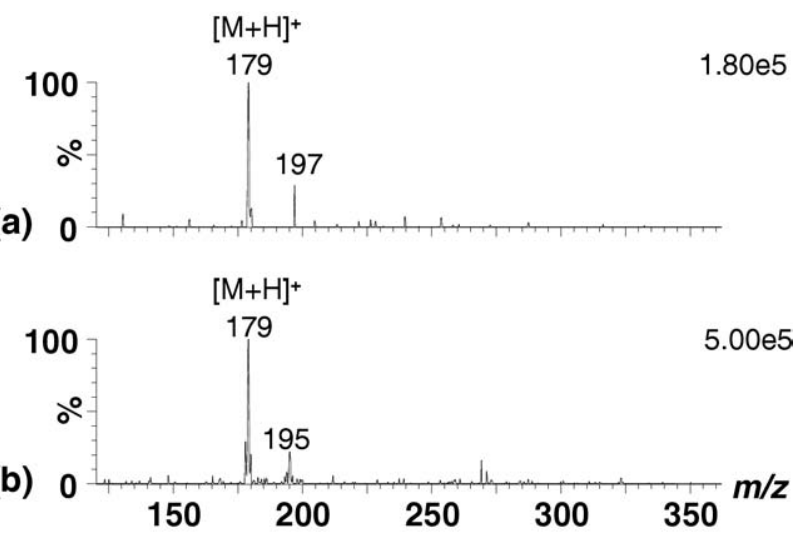

Figure 5. Mass spectrum of anthracene generated by desorption ionization by a nebulized spray of $50 \%$ methanol and $50 \%$ water containing $0.1 \%$ formic acid (a), and that with nitrogen gas only(b). The flow rate of nebulizing nitrogen was $450 \mathrm{~L} / \mathrm{h}$ for both experiments. The amount of anthracene on the target was 25 $\mathrm{ng} / \mathrm{mm}^{2}$ and the capillary voltage held at $5 \mathrm{kV}$.

ions $[16,22]$. We postulate that the mechanism of DICE is similar to that of DESI because the signal-to-noise ratios obtained for the $\mathrm{M}^{+\bullet}$ and $[\mathrm{MH}]^{+}$of anthracene, a compound of boiling point $340^{\circ} \mathrm{C}$, remained similar when nebulization gas temperature was increased from $150{ }^{\circ} \mathrm{C}$ to $450{ }^{\circ} \mathrm{C}$ (Supplementary Figure S27). Hence, the initial gaseous analyte generation mechanism by DICE is probably not a thermal desorption process.

As more information about desorption ionization mechanisms become available, it is more and more apparent that one straightforward mechanism is inadequate to account for all the features associated with a particular ionizations process. Apparently, gaseous ion formation is attributable to several mechanisms that may be taking place simultaneously. The predominant process that takes place at any microscopic location depends most likely on the physicochemical parameters that ions and molecules experience at a particular location. Our ideas about gaseous ion generation evolve as more experimental data become available. For example, chemical sputtering was one of the initial mechanisms considered for DESI [22]. Currently, momentumtransfer events are hypothesized to be the dominant process that contributes to the analyte-containing droplet formation [16]. Although, we have proposed a charge-exchange mechanism as the predominant means of ion formation in this paper, some uncertainties exists because contributions from atmosphericpressure chemical ionization related processes, depending on the chemistry of the analytes, could have added to the overall ion generation.

To optimize conditions for the DICE technique, some of the basic operating parameters were deliberately varied. A change of flow rate of the DICE reagent showed a pronounced effect on the sample peaks observed. At an infusion rate of $10 \mu \mathrm{L} / \mathrm{min}$, the intensity of the $\mathrm{m} / \mathrm{z} 179$ peak for the $[\mathrm{MH}]^{+}$was slightly higher than that $m / z 178$ for the $\mathbf{M}^{+\bullet}$ (Supplementary Figure
S-28). At higher infusion rates, on the other hand, the predominant species detected was the molecular ion (Supplementary Figure S-28A). Although an increase in baseline noise was noted at higher flow rates, the overall signal-to-noise $(\mathrm{S} / \mathrm{N})$ ratio was very high even at a flow rate of $100 \mu \mathrm{L} / \mathrm{min}$. On the other hand, ion generation by the DICE technique showed little dependence on the nebulizing gas flow rate or its temperature. In general, mass spectra of relatively high $\mathrm{S} / \mathrm{N}$ ratio can be recorded as long as the nebulizer flow rate was set above $100 \mathrm{~L} / \mathrm{h}$ (Figure S-29), and the desolvation temperature was maintained above $150{ }^{\circ} \mathrm{C}$ (Figure S-27). Most of all, the results reported here show that practically useful ion currents at an acceptable $\mathrm{S} / \mathrm{N}$ ratio can be generated from analytes by the DICE technique even under non-optimal experimental conditions.

\section{Conclusion}

A new technique for desorbing analytes from a solid surface by an atmospheric-pressure mass spectrometric technique has been developed. The spray droplets containing molecular ions are directed onto a target surface for desorption and ionization of analytes by this technique. The analyte ions generated by this technique are primarily the molecular ions produced by a charge exchange mechanism. Molecular ions from a wide range of analytes can be easily generated using this technique, which might be useful for tandem mass spectrometry since they fragment differently compared with the cationized species. DICE can easily be incorporated into an existing DESI or ESI sources, thereby, broadening the class of compounds that can be successfully analyzed by surface desorption mass spectrometry.

\section{Appendix A Supplementary Material}

Supplementary material associated with this article may be found in the online version at doi:10.1016/ j.jasms.2010.04.020.

\section{References}

1. Shiea, J.; Huang, M. Z.; Hsu, H. J.; Lee, C. Y.; Yuan, C. H.; Beech, I.; Sunner, J. Electrospray-Assisted Laser Desorption/Ionization Mass Spectrometry for Direct Ambient Analysis of Solids. Rapid Commun. Mass Spectrom. 2005, 19, 3701-3704.

2. Myung, S.; Wiseman, J. M.; Valentine, S. J.; Takats, Z.; Cooks, R. G.; Clemmer, D. E. Coupling Desorption Electrospray Ionization with Ion Mobility/Mass Spectrometry for Analysis of Protein Structures: Evidence for Desorption of Folded and Denatured States. J. Phys. Chem. B 2006, 110, 5045-5051.

3. Takats, Z.; Cotte-Rodriguez, I.; Talaty, N.; Chen, H.; Cooks, R. G. Trace Level Detection of Explosives on Ambient Surfaces by Desorption Electrospray Ionization Mass spectrometry. Chem. Commun. 2005, 15, 1950-1952.

4. Cody, R. B.; Laramee, J. A.; Durst, H. D. Versatile New Ion Source for the Analysis of Materials in Open Air under Ambient Conditions. Anal. Chem. 2005, 77, 2297-2302.

5. Nefliu, M.; Venter, A.; Cooks, R. G. Desorption Electrospray Ionization and Electrosonic Spray Ionization for Solid- and Solution-Phase Analysis of Industrial Polymers. Chem. Commun. 2006, 8, 888-890. 
6. Fernandez, F.; Cody, R. B.; Green, M. D.; Hampton, C. Y.; McGready, R.; Sengaloundeth, S.; White, N. J.; Newton, P. N. Characterization of Solid Counterfeit Drug Samples by Desorption Electrospray Ionization and Direct-Analysis-in-Real-Time Coupled to Time-of-Flight Mass Spectrometry. Chem. Med. Chem. 2006, 1, 702-705.

7. Wiseman, J. M.; Ifa, D. R.; Song, Q.; Cooks, R. G. Tissue Imaging at Atmospheric Pressure Using Desorption Electrospray Ionization (DESI) Mass Spectrometry. Angew. Chem. Int. Ed. 2006, 45, 7188-7192.

8. Morlock, G.; Ueda, Y. New Coupling of Planar Chromatography with Direct Analysis in Real Time Mass Spectrometry. J. Chromatogr. A 2007, $1143,243-251$.

9. Venter, A.; Nefliu, M.; Cooks, R. G. Ambient Desorption Ionization Mass Spectrometry. Trends Anal. Chem. 2008, 27, 284-290.

10. Haapala, M.; Pol, J.; Saarela, V.; Arvola, V.; Kotiaho, T.; Ketola, A. R.; Franssila, S.; Kauppila, J. T.; Kostiainen, R. Desorption Atmospheric Pressure Photoionization. Anal. Chem. 2007, 79, 7867-7872.

11. Wu, C.; Ifa, D. R.; Manicke, N. E.; Cooks, R. G. Rapid, Direct Analysis of Cholesterol by Charge Labeling in reactive Desorption Electrospray Ionization. Anal. Chem. 2009, 81, 7618-7624.

12. Chen, H.; Talaty, N. N.; Takats, Z.; Cooks, R. G. Desorption Electrospray Ionization Mass Spectrometry for High-Throughput Analysis of Pharmaceutical Samples in the Ambient Environment. Anal. Chem. 2005, 77, 6915-6927.

13. Wu, C.; Qian, K.; Nefliu, M.; Cooks, R. G. Ambient Analysis of Saturated Hydrocarbons Using Discharge-Induced Oxidation in Desorption Electrospray Ionization. J. Am. Soc. Mass Spectrom. 2010, 21, 261-267.
14. Van Berkel, G.; McLuckey, S. A.; Glish, G. L. Electrochemical Origin of Radical Cations Observed in Electrospray Ionization Mass Spectra. Anal. Chem. 1992, 64, 1586-1593.

15. Cotte-Rodriquez, I.; Hernandez-Soto, H.; Chen, H.; Cooks, R. G. In Situ Trace Detection of Peroxide Explosives by Desorption Electrospray Ionization and Desorption Atmospheric Pressure Chemical Ionization Anal. Chem. 2008, 80, 1512-1519S.

16. Costa, A. B.; Cooks, R. G. Simulated Splashes: Elucidating the Mechanism of Desorption Electrospray Ionization Mass Spectrometry. Chem. Phys. Lett. 2008, 464, 1-8.

17. Venter, A.; Cooks, R. G. Desorption Electrospray Ionization in a Small Pressure-Tight Enclosure. Anal. Chem. 2007, 79, 6398-6403.

18. Kebarle, P.; Tang, L. From Ions in Solution to Ions in the Gas Phase. Anal. Chem. 1993, 65, 972-986.

19. Tubaro, M.; Marotta, E.; Seraglia, R.; Traldi, P. Atmospheric Pressure Photoionization Mechanisms. 2. The Case of Benzene and Toluene. Rapid Commun. Mass Spectrom. 2003, 17, 2423-2429.

20. Cody, R. B. Observation of Molecular Ions and Analysis of Nonpolar Compounds with the Direct Analysis in Real Time Ion Source. Anal. Chem. 2009, 81, 1101-1107.

21. Hiraoka, K.; Furuya, H.; Kambara, S.; Suzuki, S.; Hasimoto, Y.; Takamizawa, A. Atmospheric-Pressure Penning Ionization of Aliphatic Hydrocarbons. Rapid Commun. Mass Spectrom. 2006, 20, 3213-3222.

22. Takats, Z.; Wiseman, J. M.; Cooks, R. G. Ambient Mass Spectrometry Using Desorption Electrospray Ionization (DESI): Instrumentation, Mechanisms, and Application in Forensics, Chemistry, and Biology. J. Mass Spectrom. 2005, 40, 1261-1275. 